UNITUE-THEP-23/2002, FAU-TP3-02/26

http://xxx.lanl.gov/abs/hep-ph/0209366

\title{
Infrared Exponents and the Running Coupling of Landau gauge QCD and their Relation to Confinement
}

\author{
R. Alkofer ${ }^{1}$, C. S. Fischer ${ }^{1}$ and L. von Smekal ${ }^{2}$ a \\ ${ }^{1}$ Institute for Theoretical Physics, University of Tübingen Auf der Morgenstelle 14, D-72076 Tübingen, Germany \\ 2 Institute for Theoretical Physics III, University of Erlangen-Nürnberg Staudtstr. 7, D-91058 Erlangen, Germany
}

September 30, 2002

\begin{abstract}
The infrared behaviour of the gluon and ghost propagators in Landau gauge QCD is reviewed. The Kugo-Ojima confinement criterion and the Gribov-Zwanziger horizon condition result from quite general properties of the ghost Dyson-Schwinger equation. The numerical solutions for the gluon and ghost propagators obtained from a truncated set of Dyson-Schwinger equations provide an explicit example for the anticipated infrared behaviour. The results are in good agreement with corresponding lattice data obtained recently. The resulting running coupling approaches a fix point in the infrared, $\alpha(0)=8.92 / N_{c}$. Two different fits for the scale dependence of the running coupling are given and discussed.
\end{abstract}

PACS. 12.38.Aw 14.70.Dj 12.38.Lg 11.15.Tk 02.30.Rz

\section{Aspects of Confinement}

Quarks and gluons, the elementary fields of QCD, are not directly detected in experiments. Instead, a plethora of hadrons, interpreted as colourless bound states, are observed. This phenomenon, called confinement, is still not properly understood, a clear and undisputable mechanism responsible for this effect has not been found yet. Moreover it seems not even clear, at present, whether the phenomenon of confinement is at all compatible with a description of quark and gluon correlations in terms of local fields in the usual sense of quantum field theory.

It is interesting to note that the two-point correlations functions of QCD, the quark, gluon and ghost propagators, might show some signals of the underlying structures of the theory which are responsible for confinement. It has been argued that the infrared behaviour of the ghost and the gluon propagator of ordinary Faddeev-Popov gauge is related to both, the Kugo-Ojima confinement criterium [1] and the Gribov-Zwanziger horizon condition [2, 33.

In the Kugo-Ojima scenario a physical state space that contains colourless states only is generated if two conditions are fulfilled: First one should not have massless particle poles in transverse gluon correlations and second one needs a well-defined, i.e. unbroken, global colour charge. The second condition can be related to the behaviour of the ghost propagator in Landau gauge. For it to be satis-

\footnotetext{
a Talk given by R.A. at the conference Quark Nuclear Physics 2002.
}

fied, the propagator must be more singular than a massless particle pole in the infrared [4].

The Gribov-Zwanziger horizon condition is connected to the gauge fixing ambiguities in the linear covariant gauge. Ideally one would eliminate Gribov copies along gauge orbits by a restriction of the functional integral of the QCD partition function to the so-called fundamental modular region. This part of configuration space lies inside the first Gribov region, a convex region in gauge field space which contains the trivial configuration $A \equiv 0$. At the boundary of the first Gribov region, the lowest eigenvalue of the Faddeev-Popov operator approaches zero. Entropy arguments have been employed to reason that the infrared modes of the gauge field are close to this Gribov horizon [3]. As the ghost propagator is the inverse of the Fadeev-Popov operator we therefore encounter the presence of the Gribov horizon in the infrared behaviour of the ghost: The ghost propagator is required to be more singular than a simple pole if the restriction to the Gribov region is correctly implemented. Furthermore, by the same entropy arguments, the gluon propagator has to vanish in the infrared [3].

Our framework to investigate the behaviour of the propagators of QCD are the Dyson-Schwinger equations (DSEs) [5]. Being complementary to lattice Monte Carlo simulations which have to deal with finite-volume effects, DSEs allow for analytical investigations of the infrared behaviour of correlation functions. In Landau gauge we have the particularly simple situation that the ghost-gluon vertex does not suffer from ultraviolet infinities. Based on 
R. Alkofer, C. S. Fischer and L. von Smekal: Infrared Exponents and Running Coupling of QCD

this observation one can use the general structure of the ghost DSE, the properties of multiplicative renormalizability and the assumption that all involved Green's functions can be expanded in a power series to show that the Kugo-Ojima criterion as well as the Gribov-Zwanziger horizon condition are satisfied [6,7]. Furthermore, it has been shown that the infrared behaviour of the ghost and the gluon propagators are uniquely related: Both fulfill power laws such that the corresponding powers in the running coupling (as extracted from the ghost-gluon vertex) exactly cancel and one obtains an infrared fix point for the coupling.

\section{Infrared exponents and running coupling}

More detailed information on the propagators of Landau gauge QCD can be obtained from the DSEs once the system is truncated and ansaetze for the vertices have been made. The resulting closed system of equations can be solved both, analytically in the infrared and numerically for non-vanishing momenta. The considerations presented in the previous section suggest that for small momenta the ghost loop dominates in the gluon DSE. Assuming this dominance, effects from a wide class of possible dressings for the ghost-gluon vertex have been investigated in ref. [7] and found to be of negligible influence to the qualitative findings.

Thus, for the purpose of this talk we concentrate on the simplest of these truncation schemes which has been developped in detail in refs. 8, 9, 9 . This scheme employs bare three-point functions and neglects four-gluon vertices, see Fig. 1. 1. It provides the correct one-loop anomalous dimensions of the ghost and gluon dressing functions, $G\left(k^{2}\right)$ and $Z\left(k^{2}\right)$, respectively, 7 and thus correctly describes the leading logarithmic behaviour of the propagators in the ultraviolet. Furthermore, this scheme reproduces the infrared exponents found in refs. [7, 11]: $Z\left(k^{2}\right) \sim\left(k^{2}\right)^{2 \kappa}$ and $G\left(k^{2}\right) \sim\left(k^{2}\right)^{-\kappa}$ with $\kappa \approx 0.595$. These exponents are close to the ones extracted from lattice calculations 12 , 13, 14. Interestingly enough they are also close to the ones obtained in a comparable truncation scheme in stochastically quantized Landau gauge Yang-Mills theory [15].

The numerical solutions are compared to recent lattice calculations [14] in Fig. 3. Differences mainly occur for the gluon propagator in the region around the bending point, i.e. somewhat below one $\mathrm{GeV}$. These can be attributed to the omission of the two-loop diagrams in the DSE truncation. Given the limitations of both methods the qualitative and partly even quantitative agreement is remarkable.

The DSE based result for the running coupling can be seen in Fig. 3. The analytically obtained value for the fix point of the running coupling in the infrared is

\footnotetext{
1 A coupled system of gluon and ghost DSEs has been studied for the first time in ref. 10].

2 These quantities are defined via the gluon and the ghost propagators via the relations $D_{\mu \nu}^{\text {Gluon }}\left(k^{2}\right)=$ $\left(\delta_{\mu \nu}-\frac{k_{\mu} k_{\nu}}{k^{2}}\right) Z\left(k^{2}\right) / k^{2}$ and $D^{\mathrm{Ghost}}\left(k^{2}\right)=-G\left(k^{2}\right) / k^{2}$.
}

$\alpha(0) \approx 2.97$ for the gauge group $\mathrm{SU}(3)$ in this truncation scheme. Corrections from possible dressings for the ghost-gluon vertex have been found to be such that $2.5<$ $\alpha(0) \leq 2.97$ [7]. The maximum at non-vanishing momenta seen in our result for the running coupling results in a multi-valued beta-function. On the other hand, it appears in a region where the above comparison to lattice data suggests that our results are least reliable. (The physical scale has been fixed by requiring the experimental value $\alpha\left(M_{Z}^{2}=(91.2 \mathrm{GeV})^{2}\right)=0.118$. $)$ We therefore summarize our result for the running coupling in the monotonic fit functions: $3^{\beta}$

$$
\begin{aligned}
\text { Fit A: } \quad \alpha(x)= & \frac{\alpha(0)}{\ln \left(e+a_{1}\left(x / \Lambda^{2}\right)^{a_{2}}+b_{1}\left(x / \Lambda^{2}\right)^{b_{2}}\right)} \\
\text { Fit B: } \quad \alpha(x)= & \frac{1}{a+\left(x / \Lambda^{2}\right)^{b}}(a \alpha(0)+ \\
& \left.\left(\frac{1}{\ln \left(x / \Lambda^{2}\right)}-\frac{1}{x / \Lambda^{2}-1}\right)\left(x / \Lambda^{2}\right)^{b}\right)
\end{aligned}
$$

The value $\alpha(0)=2.972=8.915 / N_{c}$ is known from the infrared analysis. In both fits the ultraviolet behaviour of the solution fixes the scale, $\Lambda=0.714 \mathrm{GeV}$. Note that we have employed a MOM scheme, and thus $\Lambda$ has to be interpreted as $\Lambda_{M O M}^{N_{f}=0}$, i.e. this scale has the expected magnitude. Fit A employs the four additional parameters: $a_{1}=1.106, a_{2}=2.324, b_{1}=0.004, b_{2}=3.169$. Fit $\mathrm{B}$ (which provides a better description in the ultraviolet at the expense of some deviations at smaller momenta) has only two free parameters: $a=1.020, b=1.052$.

In summary, we have employed analytical as well numerical studies of the gluon and ghost Dyson-Schwinger equations in Landau gauge Yang-Mills theories to verify the Kugo-Ojima confinement criterion. We have shown that the resulting infrared behaviour of gluon and ghost propagators, namely a highly infrared singular ghost and an infrared suppressed gluon propagator, are related to the Gribov-Zwanziger horizon condition. The solution for these propagators has then be used to calculate the running coupling for all spacelike momentum scales.

\section{Acknowledgements}

R.A. thanks the organizers of Quark Nuclear Physics 2002 for the possibility to participate in this conference. We are grateful to K. Langfeld, H. Reinhardt, D. Shirkov, P. Watson and D. Zwanziger for helpful discussions.

This work has been supported by the DFG under contract $\mathrm{Al} 279 / 3-4$ and by the European graduate school Tübingen-Basel (DFG contract GRK 683).

\section{References}

1. T. Kugo and I. Ojima, Prog. Theor. Phys. Suppl. 66 (1979) 1 .

${ }^{3}$ The $\beta$-function corresponding to fit $\mathrm{A}$ can be found in ref. 16. 
R. Alkofer, C. S. Fischer and L. von Smekal: Infrared Exponents and Running Coupling of QCD

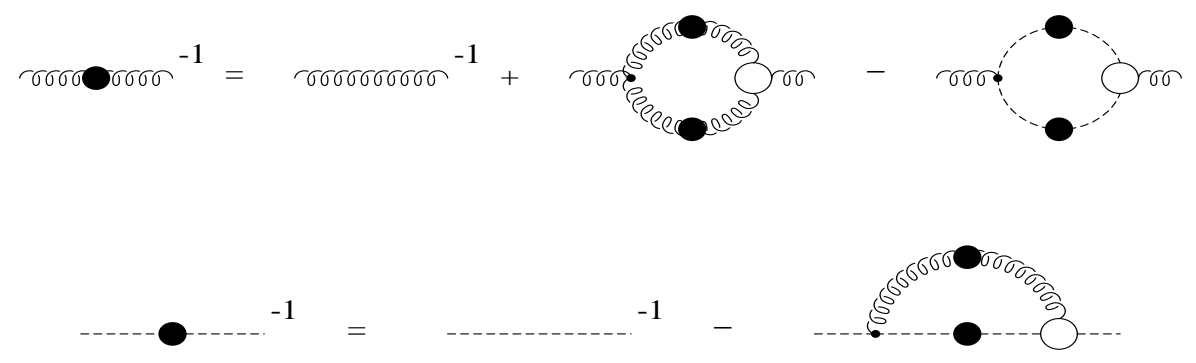

Fig. 1. Diagrammatic representation of the truncated gluon and ghost Dyson-Schwinger equations (DSE). Terms with fourgluon vertices have been dismissed. Herein, the vertex functions (empty circles) are taken to be the bare vertices.

DSE vs. lattice results $\left(16^{3} \times 32\right)$

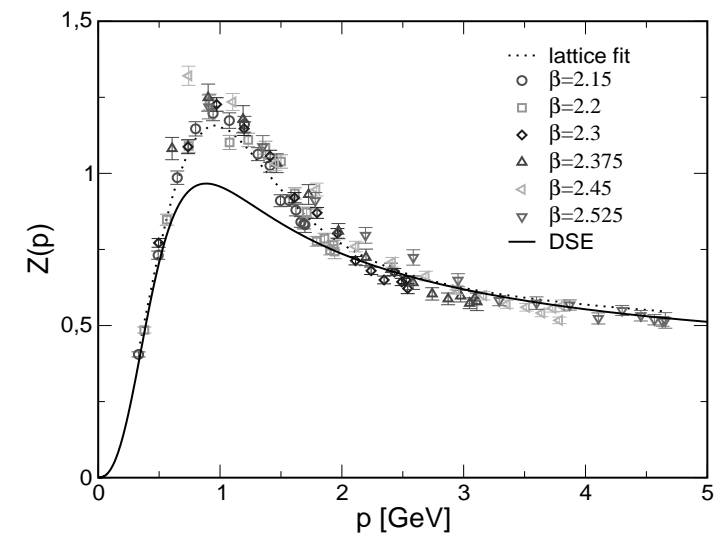

DSE vs. lattice results $\left(16^{3} \times 32\right)$

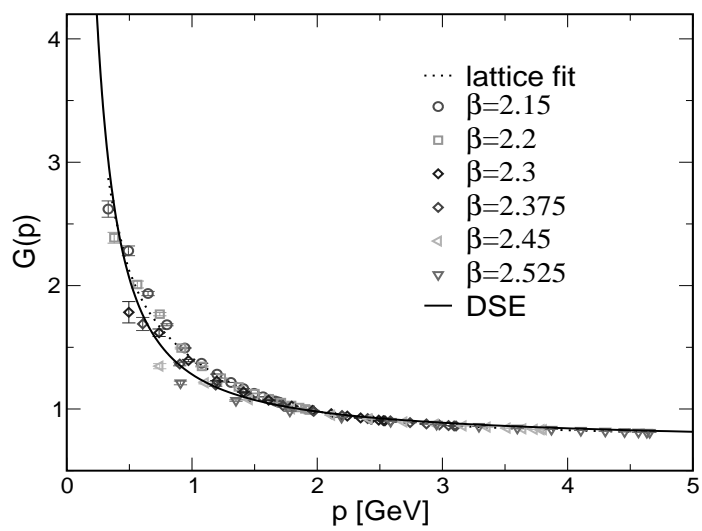

Fig. 2. Solutions of the Dyson-Schwinger equations compared to recent lattice results for two colours 14

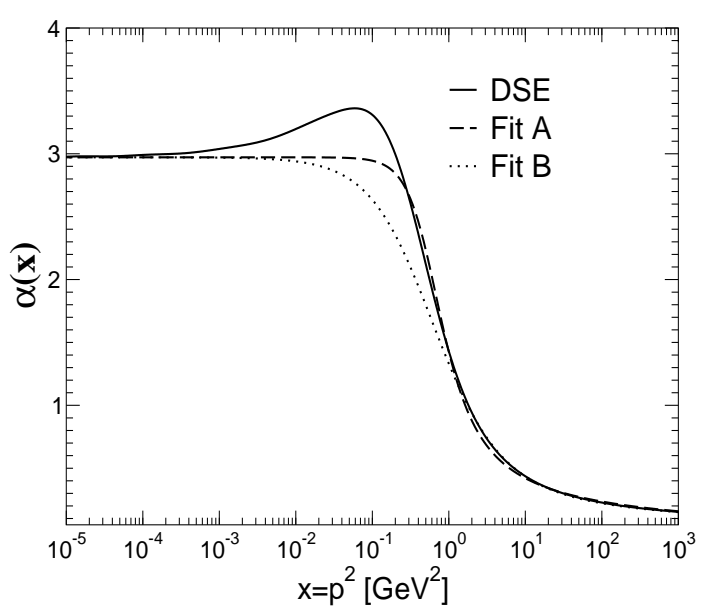

Fig. 3. The strong running coupling from the DSEs and the fits $\mathrm{A}$ and B, c.f. eqs. (国, 昍).

2. V. N. Gribov, Nucl. Phys. B 139 (1978) 1.

3. D. Zwanziger, Nucl. Phys. B 364 (1991) 127; Nucl. Phys. B 399 (1993) 477; Nucl. Phys. B 412 (1994) 657.

4. T. Kugo, Int. Symp. on BRS symmetry, Kyoto, Sep. 18-22, 1995, arXiv:hep-th/9511033.

5. R. Alkofer and L. von Smekal, Phys. Rept. 353 (2001) 281 arXiv:hep-ph/0007355.
6. P. Watson and R. Alkofer, Phys. Rev. Lett. 86 (2001) 5239 arXiv:hep-ph/0102332;

R. Alkofer, L. von Smekal and P. Watson, Proceedings of the ECT* Collaboration Meeting on Dynamical Aspects of the QCD Phase Transition, Trento, Italy, March 12-15, 2001, arXiv:hep-ph/0105142.

7. C. Lerche and L. von Smekal, Phys. Rev. D 65 (2002) 125006 arXiv:hep-ph/0202194.

8. C. S. Fischer, R. Alkofer and H. Reinhardt, Phys. Rev. D 65 (2002) 094008 arXiv:hep-ph/0202195.

9. C. S. Fischer and R. Alkofer, Phys. Lett. B 536 (2002) 177 arXiv:hep-ph/0202202.

10. L. von Smekal, R. Alkofer and A. Hauck, Phys. Rev. Lett. 79 (1997) 3591 arXiv:hep-ph/9705242]; Annals Phys. 267 (1998) 1 arXiv:hep-ph/9707327.

11. D. Zwanziger, Phys. Rev. D 65 (2002) 094039 arXiv:hepth/0109224.

12. F. D. Bonnet, P. O. Bowman, D. B. Leinweber and A. G. Williams, Phys. Rev. D 62 (2000) 051501 arXiv:heplat/0002020.

13. F. D. Bonnet, P. O. Bowman, D. B. Leinweber, A. G. Williams and J. M. Zanotti, Phys. Rev. D 64 (2001) 034501 arXiv:hep-lat/0101013.

14. K. Langfeld, H. Reinhardt and J. Gattnar, Nucl. Phys. B 621 (2002) 131 arXiv:hep-ph/0107141:

see also: K. Langfeld et al. arXiv:hep-th/0209173.

15. D. Zwanziger, arXiv:hep-th/0206053.

16. R. Alkofer, C. S. Fischer and L. von Smekal, Acta Phys. Slov. 52 (2002) 191 arXiv:hep-ph/0205125. 